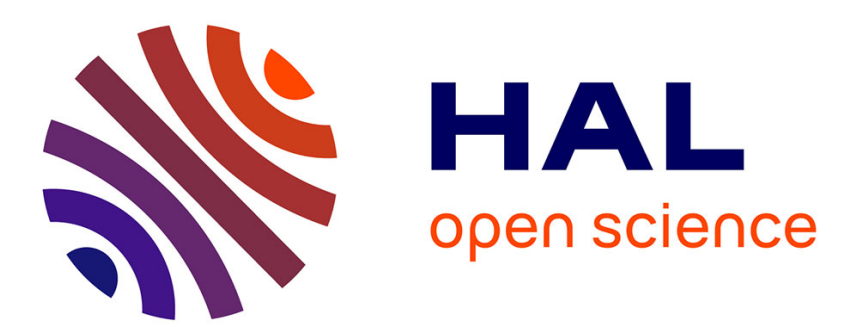

\title{
Numerical study of scratch velocity effect on recovery of viscoelastic-viscoplastic solids
}

Nicolas Aleksy, Guillaume Kermouche, Alain Vautrin, Jean-Michel Bergheau

\section{To cite this version:}

Nicolas Aleksy, Guillaume Kermouche, Alain Vautrin, Jean-Michel Bergheau. Numerical study of scratch velocity effect on recovery of viscoelastic-viscoplastic solids. International Journal of Mechanical Sciences, 2010, 52 (3), pp.455-463. 10.1016/j.ijmecsci.2009.11.006 . hal-00850009

\section{HAL Id: hal-00850009 \\ https://hal.science/hal-00850009}

Submitted on 10 Dec 2019

HAL is a multi-disciplinary open access archive for the deposit and dissemination of scientific research documents, whether they are published or not. The documents may come from teaching and research institutions in France or abroad, or from public or private research centers.
L'archive ouverte pluridisciplinaire HAL, est destinée au dépôt et à la diffusion de documents scientifiques de niveau recherche, publiés ou non, émanant des établissements d'enseignement et de recherche français ou étrangers, des laboratoires publics ou privés. 


\title{
Numerical study of scratch velocity effect on recovery of viscoelastic-viscoplastic solids
}

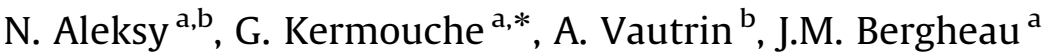 \\ a Université de Lyon, ENISE, LTDS UMR 5513 CNRS, 58 rue J. Parot, 42023 Saint-Etienne Cedex 2, France \\ ${ }^{\mathrm{b}}$ Université de Lyon, ENSM-SE, LTDS UMR 5513 CNRS, 158 cours Fauriel, 42023 Saint-Etienne Cedex 2, France
}

\begin{abstract}
The scratch test is a classical way to investigate the abrasive resistance of coatings and substrates. Because of the complex phenomena involved, the use of refined finite element analysis is often required to analyze the influence of specific parameters. In this paper, the influence of the tip velocity on the scratch recovery of polymer-like time-dependent solids is qualitatively investigated. More precisely the response of three constitutive models is analyzed: an elastic-viscoplastic model, a linear viscoelastic model and finally a viscoelastic-viscoplastic model. This last model is an original assembly based on the connection in series of the elastic-viscoplastic model and the linear viscoelastic model. For that, a new method allowing the connection in series of two different rheological models in a FE code is presented. To analyze the numerical results, the concept of representative stress and representative strain rate of a scratch test is introduced.
\end{abstract}

\section{Introduction}

The scratch loading is a classical way to characterize the abrasive resistance of engineering surfaces such as coating over substrate systems.

In a standard single pass scratch test, a hard indenter slides along the surface of a softer material. The induced normal and tangential forces as well as the resulting scratch morphology are analyzed. Three main phenomena can be observed during a scratch test: ploughing which induces ridges in front and on sides of indenter; the cutting which leads to material removal due to the formation of a chip in front of the indenter; cracking leading to cracks formation and propagation. In this paper only the scratch-induced deformation will be investigated which corresponds to the ploughing phenomenon.

Because of the high number of parameters involved in the experimental tests, the analysis of the scratch test is really a difficult and expensive task. The finite element method can be used to overcome these difficulties [1-3]. Indeed, the influence of each parameter can be studied separately in order to achieve a better understanding of the scratch test.

During the last decade, the scratch test of time-dependent solids has been widely investigated, due to the intensive use of amorphous polymers in a large number of industrial sectors [4]. In particular, research in optics is currently focusing on light glasses

\footnotetext{
* Corresponding author. Tel.: +3347743 7531; fax: +33477437539.

E-mail address: guillaume.kermouche@enise.fr (G. Kermouche).
}

in polymers which are more comfortable but also more sensitive to daily aggressions $[5,6]$. Hence, the glass surface protection is usually ensured by thin coating films. Amorphous polymers are the main constituting materials of these layers. As scratching is one of the major stresses for such a surface, specific studies have to be performed.

This paper mainly focus on the influence of the representative strain rate on the material recovery after scratching. More precisely the response of three constitutive models is analyzed: an elastic-viscoplastic model, a linear viscoelastic model and finally a viscoelastic-viscoplastic model. To analyze the numerical results, the concept of representative stress and representative strain rate of a scratch test is used.

\section{The scratch recovery}

The ploughing phenomenon is a rapidly achieved steady state regime. It can be viewed as a permanent material flow around a rigid obstacle. Theoretically, this steady state regime can be achieved under constant imposed normal force or constant imposed penetration depth of the indenter [7]. From a practical point of view, the way the loading is monitored do not matter as long as only the steady state regime is studied. Most of the experimental devices allows the measure of the normal and tangential forces [8-10] during the scratch. The scratch-induced deformation is often measured after the scratch and so only the residual print is used to perform the analysis. It is the reason why 
we only focus on the residual morphology after scratching in this paper. More specifically, the scratch-induced deformation response of materials is often evaluated as their capability to recover. Two kinds of recovery can be distinguished:

- The elastic recovery, which refers to the instantaneous elastic response of solids.

- The viscoelastic recovery, which refers to the delayed elastic response of time-dependent solids. In this case the value of the recovery depends directly on the instant where the measure is done.

\subsection{Definition}

For a time-dependent viscoelastic material, it is really difficult to separate the respective parts of elastic and viscoelastic recovery at a given time. Therefore we propose to call "scratch recovery", the following quantity [11]:

$\mathfrak{R}=1-\frac{h_{p}}{h}$

where $h_{p}$ is the depth of the residual groove measured in the steady-state regime and $h$ is the penetration depth of the indenter (Fig. 1). Let us note that the measure is performed $10000 \mathrm{~s}$ after scratching in our numerical simulations to be sure that all relaxation processes are over. From a phenomenological point of view, this parameter represents the reversible part of the strain compared to the total strain.

When the scratch recovery $\mathfrak{R}$ is equal to 1 , the residual groove on the surface behind the indenter vanishes totally. Hence the scratch is purely reversible, instantaneous (elastic) or not (viscoelastic). For value of $\mathfrak{R}$ equals to 0 , the scratch is purely plastic. For metals, the scratch recovery is negligible, and consequently $h_{p}$ is close to $h$, whereas for polymer the scratch recovery can be much more important [12].

\subsection{Experimental evidences}

Many experimental studies have been carried out on the deformation of amorphous polymers during scratch tests $[8,9]$. Most of them have concluded on the strong influence of the tip velocity and they have linked this phenomenon to the timedependent properties of the materials. More precisely, experiments carried out by Gauthier et al. [6,13] on PMMA (polymethylmethacrylate) with a spherical hard indenter have shown that the higher the tip velocity, the higher the recovery. Different values of the load and the tip radii have been used. In their experimental conditions, they have shown that the PMMA behavior moves from elasticity at the highest tip velocity $\left(0.71 \mathrm{~mm} \mathrm{~s}^{-1}\right)$ to viscoelasticity at a lower velocity and finally to elastic-viscoplasticity at the lowest velocity $\left(0.001 \mathrm{~mm} \mathrm{~s}^{-1}\right)$. The reversibility of the strain induced by the scratch test on such a material is clearly shown to be dependent on the tip velocity.

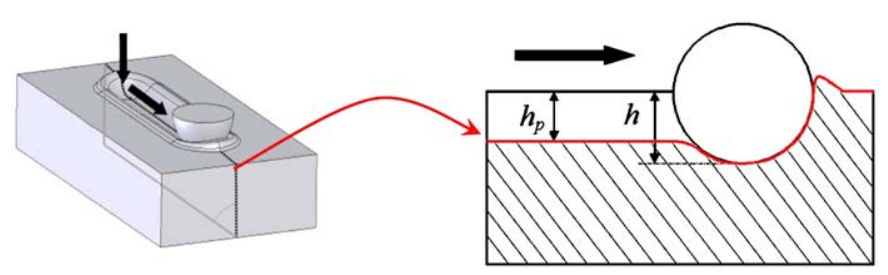

Fig. 1. Side view of the scratch.
Moreover, one major conclusion is that both viscoelastic and viscoplastic phenomena have to be accounted for to analyze scratch data. These experiments have been carried out using a constant normal load applied on the indenter.

\section{Constitutive models}

Amorphous polymers are well known for their complex mechanical behavior, which strongly depends on the thermomechanical history [14]. In this paper, temperature dependency is not accounted for. The phenomenological study here only focuses on the time dependency. Such materials exhibit a viscoelastic behavior at small strain. However, at high strain level, they present an elastic-viscoplastic behavior combined with an exponential strain hardening [14]. Consequently, glassy polymers are sometime considered as viscoelastic-viscoplastic solids $[15,16]$. These rheological models lead to classical stress/strain curves given in Figs. 2-4.

In this context, we propose in this paper the study of the influence of each of these three main mechanical behaviors on the scratch response. The ploughing process involves plastic strain, consequently the modeling of the viscoplasticity needs the use of specifically dedicated behavior law. We have chosen the elastic viscoplastic model of Arruda and Boyce $[17,18]$. However, before the performing of a specific study dealing with the real necessity (or not) to account for the viscoelastic behavior of the amorphous polymer in scratch test, we have to understand the influence of such behavior. Therefore, the viscoelastic model of Poynting is considered in this paper. Its parameters are not directly identified on a polymer because it is not necessary for this phenomenological study. Finally a viscoelastic viscoplastic model is build up thanks to a specific algorithm allowing to connect in series two models of any kind.

\subsection{The viscoplastic model}

To model the viscoplastic behavior of amorphous polymers, the Arruda-Boyce model [17,18], modified by Hasan [19], is chosen. It is composed of a Argon viscosity [20], in parallel with a Langevin rubbery hyperelasticity. This element, used to model the strain hardening, is not accounted for in our study because we have observed that if the scratch severity does not exceed $a_{c} / R=0.4$ (contact radius on tip radius), the strain level is not high enough to activate this element. Finally a linear elasticity is added in series in order to model the behavior at small strain (Fig. 5). Scalar equation of the Argon viscous element is written as

$\dot{\gamma}^{p}=\dot{\gamma}_{0} \exp \left[\frac{A}{k \theta}(\tau-s)\right]$

where $\dot{\gamma}^{p}$ represents the plastic shear strain rate, $\dot{\gamma}_{0}$ is a factor proportional to the attempt frequency, $s=0.077 G /(1-v)$ is the athermal shear strength, $G$ is the elastic shear modulus, $A$ is the zero stress level activation energy, $\tau$ is the effective equivalent shear strength, $k$ is Boltzmann's constant, $\theta$ is absolute temperature. The parameters are given in Table 1 . The value of the Young modulus $E_{2}$ is given in the following section.

\subsection{The viscoelastic model}

The viscoelastic constitutive model is based on the Poynting model [21]. It consists of a linear elastic spring in series with the parallel combination of a linear elastic spring and a deviatoric viscous dashpot $\left(\sigma=\eta_{1} \dot{\varepsilon}\right)$ (Fig. 6). This model allows to reproduce creep and relaxation phenomena. Let us note that the present model is developed in a three dimensional configuration. 


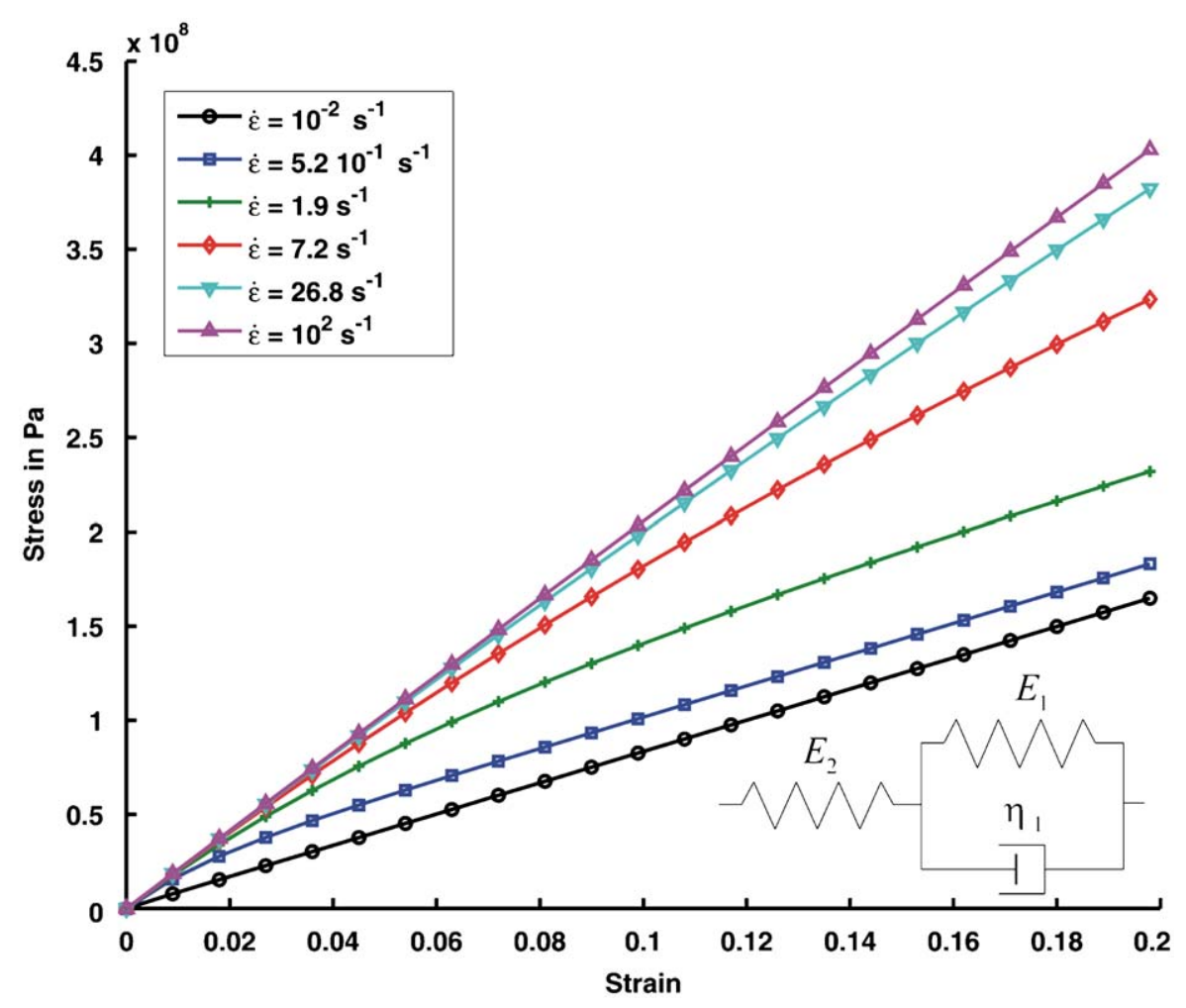

Fig. 2. Stress-strain curve for a viscoelastic model at different strain rates.

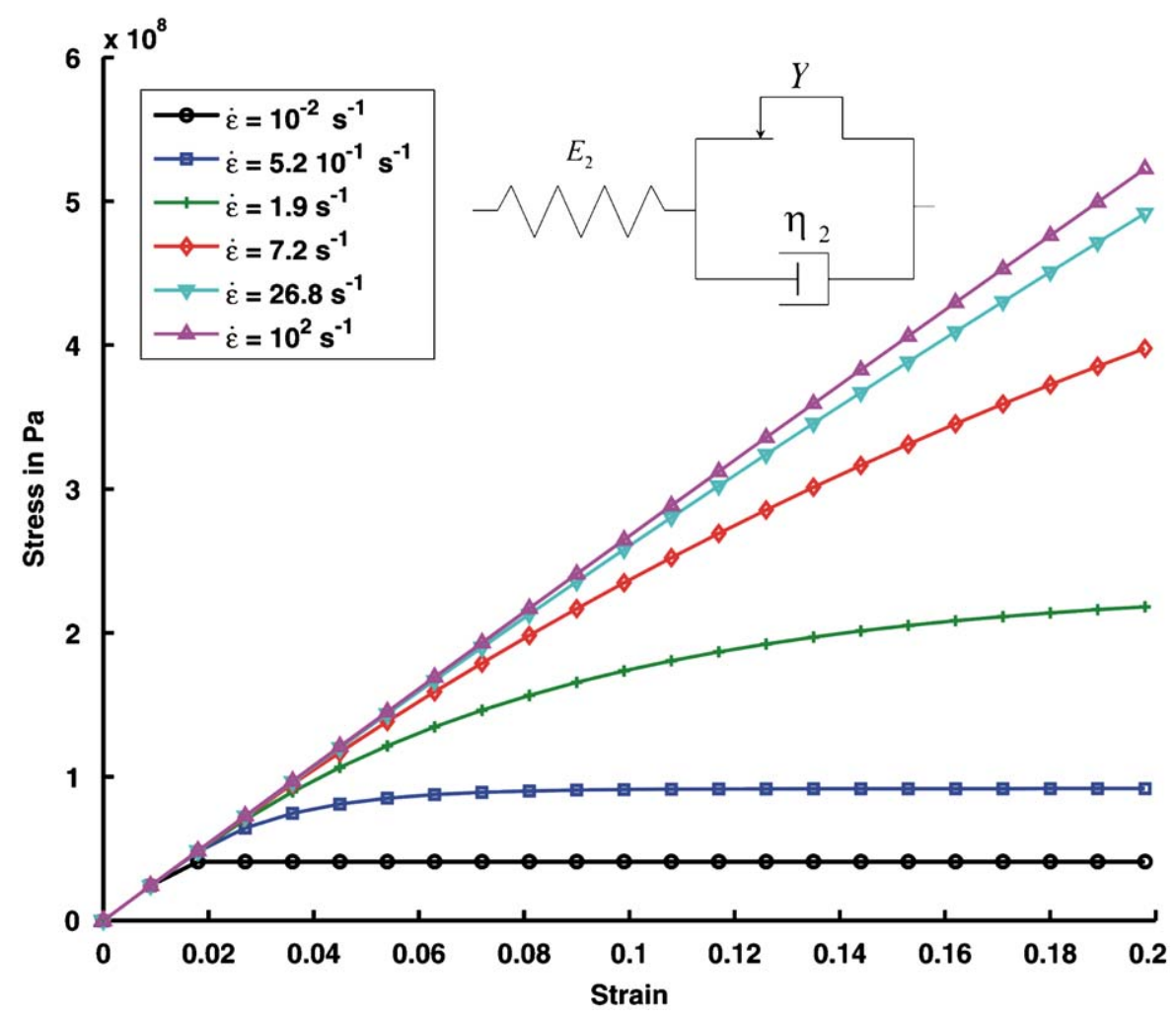

Fig. 3. Stress-strain curves for an elastic-viscoplastic model at different strain rates.

Consequently, the stiffness modulus can be expressed as the additive composition of the bulk modulus $K$ and the shear modulus $G(1 / E=1 / 9 K+1 / 3 G)$. Since the viscous element of this Poynting model follows a deviatoric definition, only the shear modulus $G$ is affected by the strain rate, and not $K$. Nevertheless, the equivalent moduli can be calculated and for the two limit 


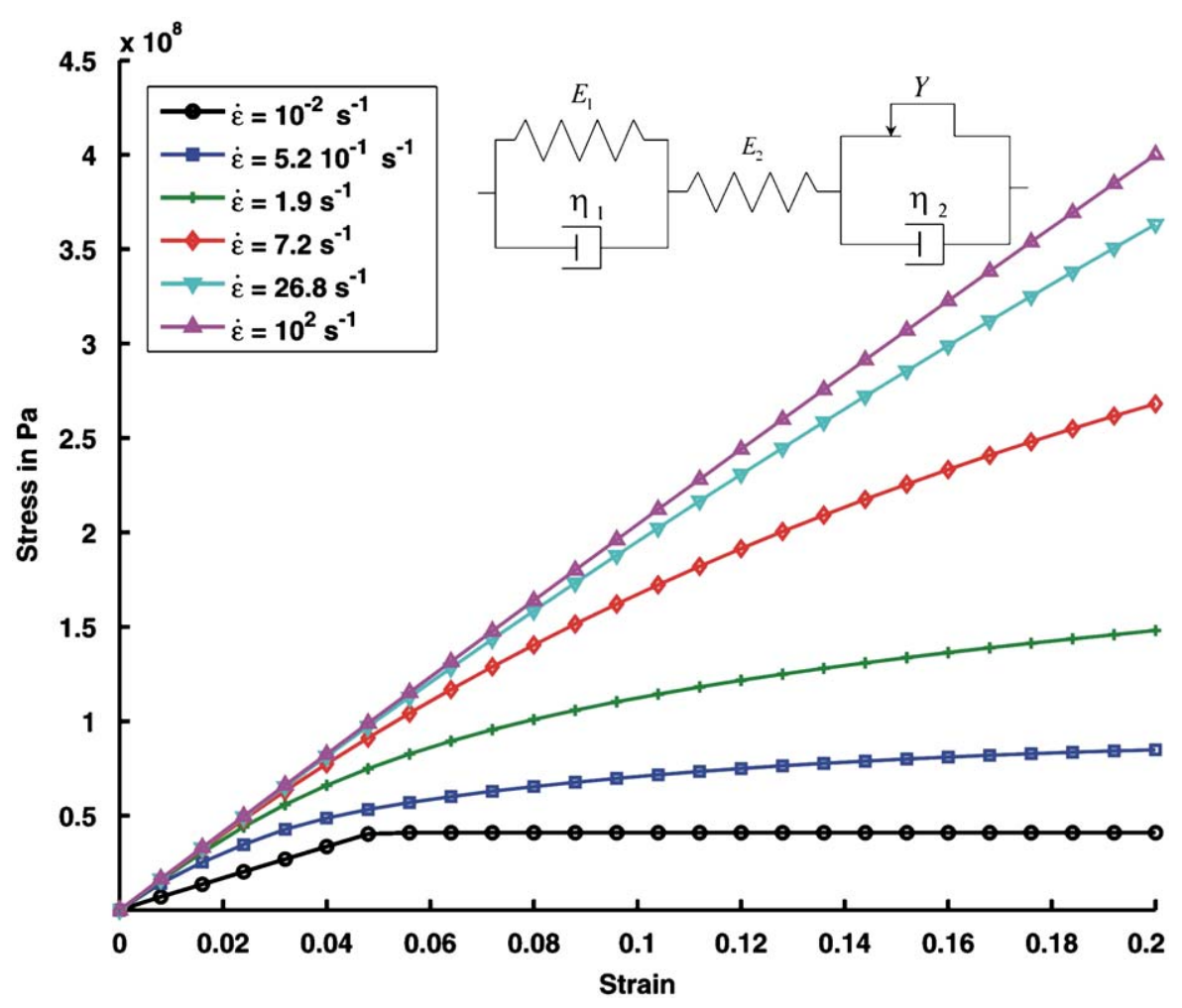

Fig. 4. Stress-strain curve for a viscoelastic-viscoplastic model at different strain rates.

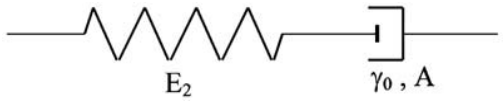

Fig. 5. Arruda-Boyce model without its strain hardening element.

Table 1

Parameters of the elastic-viscoplastic model of Arruda-Boyce.

\begin{tabular}{llll}
\hline$v$ & $\dot{\gamma}_{0}\left(\mathrm{~s}^{-1}\right)$ & $A\left(\mathrm{~m}^{3}\right)$ & $\theta(\mathrm{K})$ \\
\hline 0.33 & $2.8 \times 10^{7}$ & $1.39 \times 10^{-27}$ & 298 \\
\hline
\end{tabular}

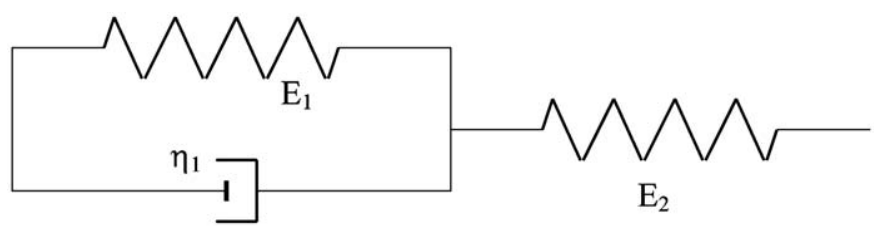

Fig. 6. Rheological representation of the Poynting model.

states, the shear modulus is computed as follows:

$\frac{1}{G_{e q}^{0}}=\frac{1}{G_{1}}+\frac{1}{G_{2}}$

$G_{e q}^{\infty}=G_{2}$

From these considerations, the Poisson ratio is affected by the strain rate as well. The values of the parameters have been chosen in relation with those of the PMMA. Since this study aims at a phenomenological approach of the time-dependent scratch response of solids, the viscous parameter $\eta_{1}$ has been chosen to observe the viscoelastic transition in the middle of the tip velocities range. The whole parameters are given in Table 2 .
Table 2

Parameters of the viscoelastic model of Poynting.

\begin{tabular}{llll}
\hline$E_{1}(\mathrm{MPa})$ & $E_{2}(\mathrm{MPa})$ & $v_{1}=v_{2}=v$ & $\eta_{1}\left(\mathrm{MPa} \mathrm{s}^{-1}\right)$ \\
\hline 785 & 2730 & 0.33 & 150 \\
\hline
\end{tabular}

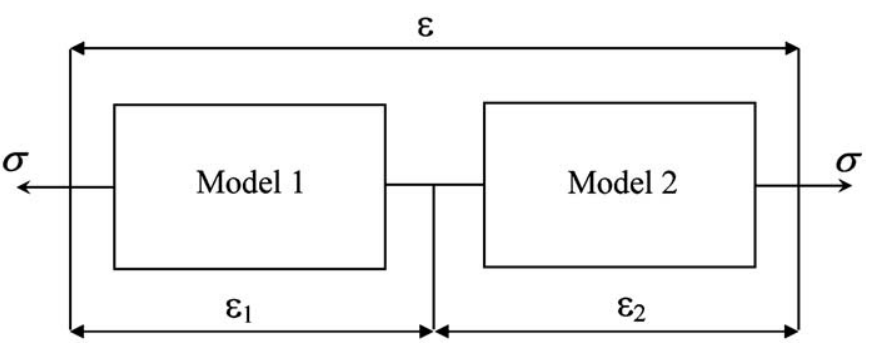

Fig. 7. Series assembly of two rheological models.

3.3. The viscoelastic viscoplastic model thanks to the "series model"

To account for both viscoelasticity and viscoplasticity we have developed in the finite element code Systus ${ }^{\circledR}$ [22] a specific algorithm, named "series model", which allows to combine in series two models of any kind (Fig. 7). Such an algorithm is helpful to combine very quickly whatever models since their constitutive equations are available in the finite element code. Our case study needs to connect a viscoelastic model with a viscoplastic one.

\subsubsection{Hypotheses}

The algorithm is here described in an updated Lagrangian formulation by using the relationship between the Cauchy stress tensor $\boldsymbol{\sigma}$ and the Eulerian strain rate tensor $\boldsymbol{D}$. Hence it can be 


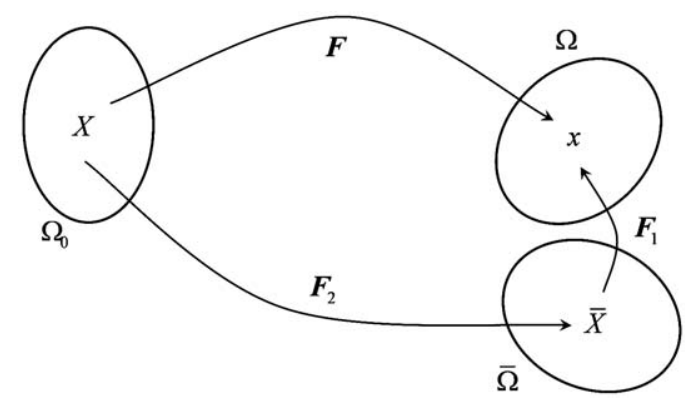

Fig. 8. Decomposition of deformation gradient via a intermediate virtual configuration.

used in either Lagrangian actualized formulation or quasi-Euler formulation.

Moreover the series model is developed in the general case (finite transformation), and so it is based on the multiplicative decomposition of the deformation tensor $\boldsymbol{F}$ [23]:

$\boldsymbol{F}=\boldsymbol{F}_{\mathbf{1}} \cdot \boldsymbol{F}_{\mathbf{2}}$

where $\boldsymbol{F}_{\mathbf{1}}$ refers to the deformation tensor for the model 1 and $\boldsymbol{F}_{\mathbf{2}}$ to the model 2 (Fig. 8).

The major assumption of this algorithm takes place here. The main problem in the numerical implementation of this algorithm is that the finite element code needs a direct relationship between the strain rate taking place in the model $1, \boldsymbol{D}_{1}$, and the one in the model 2, $\boldsymbol{D}_{\mathbf{2}}$. We propose that one of the assembled models (model 1) gives very small deformation compared to the other (Fig. 8). That is to say that the deformation in the model 1 is almost equal to the identity tensor:

$F_{1} \approx I d$

Thanks to this helpful assumption, the Eulerian strain rate tensor, $\boldsymbol{D}$, can be written as the additive decomposition of the strain rate tensor from the model $1, D_{1}$, and the one from the model 2, $\boldsymbol{D}_{\mathbf{2}}$ :

$D=D_{1}+D_{2}$

\subsubsection{Algorithm}

At each integration point of each element of the mesh and for each time step, the previous decomposition of the strain rate tensor $\boldsymbol{D}$ is assumed (Eq. (6)). As the two models are connected in series, one has:

$\sigma=\sigma_{1}=\sigma_{2}$

where $\sigma, \sigma_{1}$ and $\sigma_{2}$ are, respectively, the total stress tensor, stress tensor in the model 1, and the one in the model 2 (Fig. 7).

Starting from a known configuration at time $t$ one has to find the stress tensor at time $t+\Delta t$ solving:

$\sigma(t+\Delta t)=\sigma_{1}(t+\Delta t)=\sigma_{2}(t+\Delta t)$

Since the aim of this algorithm is to connect two models available in the finite element code, the solutions of their constitutive equations are obtained from dedicated routines and can be written as

Model 1 : $\quad \boldsymbol{\sigma}_{1}=\wp_{1}\left(\boldsymbol{D}_{1}, \boldsymbol{X}_{1}, t, \ldots\right)$

Model 2: $\quad \boldsymbol{\sigma}_{\mathbf{2}}=\wp_{2}\left(\boldsymbol{D}_{\mathbf{2}}, \boldsymbol{X}_{\mathbf{2}}, t, \ldots\right)$

Furthermore, the finite element code provides, at each time step and each integration point, the total strain rate tensor, $\boldsymbol{D}$, computed from the displacement at time $t+\Delta t$. Consequently the unknown quantities at $t+\Delta t$ are :

$\sigma(t+\Delta t)$
$\sigma_{1}(\boldsymbol{t}+\Delta t)$ and $\quad \sigma_{2}(t+\Delta t)$

$D_{1}(t+\Delta t) \quad$ and $\quad D_{2}(t+\Delta t)$

All the quantities at the previous time step have been calculated and are thus perfectly known. The series model has to calculate, and provide to the code, the total stress tensor, $\sigma$, at $t+\Delta t$ satisfying Eq. (8). The algorithm uses an iterative process which calculates the repartition of the strain rate to ensure the equality of the stress tensors (Eq. (9)).

To simplify the notation, the value at $t+\Delta t$ of any quantity $A$ noted $A(t+\Delta t)$ is for the sequel of this section written as $A$.

At iteration $i$, the algorithm calculates the stress tensors $\boldsymbol{\sigma}_{\mathbf{1}}^{i}$ and $\boldsymbol{\sigma}_{\mathbf{2}}^{i}$ from the strain rates $\boldsymbol{D}_{\mathbf{1}}^{i}$ and $\boldsymbol{D}_{\mathbf{2}}^{i}$ according to Eq. (9):

$\boldsymbol{\sigma}_{\mathbf{1}}^{i}=\wp_{1}\left(\boldsymbol{D}_{\mathbf{1}}^{i}, \Delta t, \ldots\right)$

$\boldsymbol{\sigma}_{\mathbf{2}}^{i}=\wp_{2}\left(\boldsymbol{D}_{\mathbf{2}}^{i}, \Delta t, \ldots\right)$

A convergence criterion is then applied:

$\frac{\left(\boldsymbol{\sigma}_{\mathbf{1}}^{i}-\boldsymbol{\sigma}_{\mathbf{2}}^{i}\right)_{e q}}{\sigma_{e q 1}^{i}+\sigma_{e q 2}^{i}}<\alpha$

where $\alpha$ corresponds to the prescribed precision value and $A_{e q}$ is the quadratic norm of $A$ :

$A_{e q}=(\boldsymbol{A}: \boldsymbol{A})^{1 / 2}$

If the criterion is not satisfied, the strain rates are adjusted from a first order development of the quantity $\boldsymbol{\sigma}_{\mathbf{2}}^{i}-\boldsymbol{\sigma}_{\mathbf{1}}^{i}$ :

$\left[\frac{d\left(\boldsymbol{\sigma}_{\mathbf{2}}-\boldsymbol{\sigma}_{\mathbf{1}}\right)}{d \boldsymbol{D}_{\mathbf{1}}}\right]^{i}\left\{\boldsymbol{D}_{\mathbf{1}}^{i+1}-\boldsymbol{D}_{\mathbf{1}}^{i}\right\}+\left\{\boldsymbol{\sigma}_{\mathbf{2}}^{i}-\boldsymbol{\sigma}_{\mathbf{1}}^{i}\right\}=\{\mathbf{0}\}$

This algorithm is summarized as in Fig. 9.

\subsubsection{The viscoelastic viscoplastic model}

In our case study, the model 1 refers to the Kelvin-Voigt model and the model 2 corresponds to the Arruda-Boyce model previously presented (Fig. 10). The viscoelastic response of such association is due to the Kelvin-Voigt model plus the elastic element of the Arruda-Boyce model which constitute the Poynting model already presented. Consequently the parameters of this viscoelastic part come from the Poynting model, whereas the viscoplastic ones refer to the viscous element of the ArrudaBoyce model.

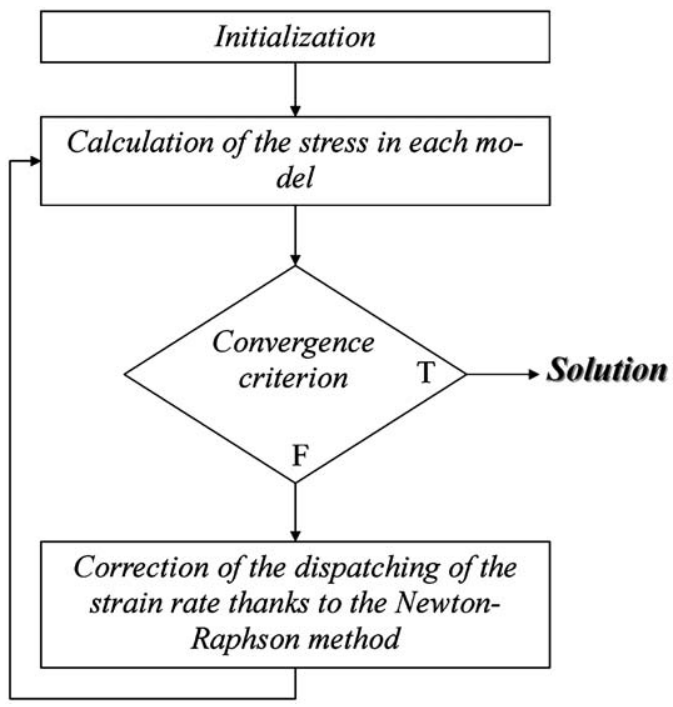

Fig. 9. Series model algorithm. 


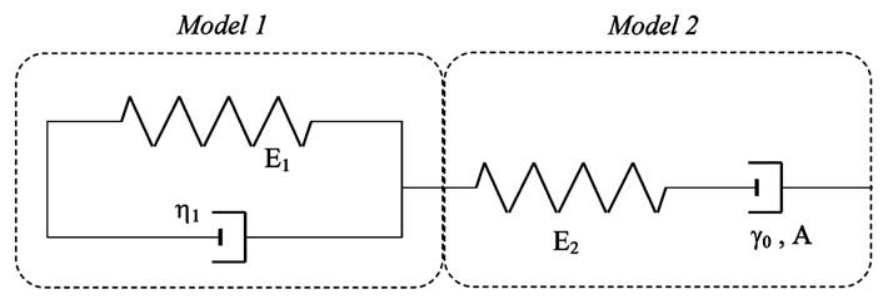

Fig. 10. Viscoelastic-viscoplastic model thanks to the series model.

\section{Computational modeling of a scratch test}

The loading is achieved by adjusting the quasi-static displacement of the indenter which is first pushed down vertically into the specimen and then moved horizontally to realize the ploughing. In this approach, the indenter is subjected to imposed vertical and horizontal displacements. The scratch distance has to be sufficiently long to reach the steady state. The finite element domain is a right-angled parallelepiped. The $x$-axis and $z$-axis are, respectively, the scratch and indentation axes. The plane $y=0$ is a symmetry plane. The mesh is constituted of 8-node-brick elements. The numerical simulations are performed using a large displacement/large strain option (updated Lagrangian formulation). The indenter is a rigid sphere of $1 \mathrm{~mm}$ radius and its penetration depth is $100 \mu \mathrm{m}$. The scratch test is performed without the use of a remeshing algorithm because the material is assumed as relatively soft and the contact frictionless. Let us recall here that a special remeshing algorithm dedicated to the scratch test of bilayer material has been recently developed in the FEM software Systus ${ }^{\circledR}$ package $[7,10]$ and thus allows to perform such types of simulations.

\section{Phenomenological study}

\subsection{Scratch recovery and representative strain rate}

As suggested by many authors [24,8,2,12,25], the scratchinduced deformation of solids can be qualitatively explained using the concept of representative parameters. Let us note that this concept has been preliminarily used with success on indentation experiments [24,26-29].

The representative stress is often related to the mean contact pressure:

$\sigma_{r} \propto \frac{F_{n}}{\pi a_{c}^{2}}$

where $F_{n}$ is the normal load and $a_{c}$ the contact radius. Let us note that the representative stress of scratch experiments performed with the same normal load is not constant because the contact radius is a function of the material behaviors and the scratch velocity. As shown by Briscoe et al. [8], Gauthier et al. [6], the scratch-induced deformation of time-dependent solids is strongly related to the representative strain rate defined by :

$\dot{\varepsilon}_{r} \propto \frac{V}{a_{c}}$

where $V$ is the tip velocity. Hence the higher the tip velocity $V$, the higher the representative strain rate $\dot{\varepsilon}_{r}$.

The representative strain of scratch experiments is related to the contact severity. For a rigid spherical indenter, it is often written as:

$\varepsilon_{r} \propto \frac{a_{c}}{R}$ where $R$ is the ball radius. For such a tip, the higher the penetration depth $h$, the higher the contact radius and thus higher the representative strain. In this paper, each scratch test is performed with the same penetration depth, thus the representative strain is approximately constant. The representative strain can be shared into an elastic representative strain (reversible part) and a plastic representative strain (irreversible part):

$\varepsilon_{r}=\varepsilon_{r}^{e}+\varepsilon_{r}^{p}$

From a phenomenological point of view, the scratch recovery corresponds to the ratio of reversible strain over the total strain. The representative strain being constant in the following numerical simulations, the scratch recovery will only be a function of the elastic representative strain. This quantity can be simply approximated by:

$\varepsilon_{r}^{e}=\frac{\sigma_{r}}{E}$

Hence it follows:

$\mathfrak{R} \propto \frac{\varepsilon_{r}^{e}}{\varepsilon_{r}} \propto \frac{\sigma_{r} / E}{a_{c} / R}$

\subsection{Elastic-viscoplastic scratching}

Because of its sensitivity to the strain rate, using a viscoplastic model allows to analyze the time effects on the scratch response. Since the simulations are performed at a constant penetration depth the contact radius is also considered as constant. Consequently, a variation of the scratch velocity leads directly to a variation of the strain rate.

The selected model is the modified Arruda-Boyce model from the previous section (Fig. 5). The value of the Young modulus is not taken from Ref. [17] but from the viscoelastic limits $E_{e q}^{\infty}$ and $E_{e q}^{0}$ (see Section 3). Hence two different series of numerical simulations have been carried out: one with $E_{e q}^{0}$ and one with $E_{e q}^{\infty}$. The aim is not to predict exactly the behavior of the PMMA but rather to show the effect of such modeling on the scratch response.

Fig. 11 shows the variation of the recovery with the tip velocity. It can be seen that the recovery increases with the tip velocity for both cases that is to say that the reversible part of the strain increases with the tip velocity. It can be explained like that: because of the viscous element of the model, the representative stress is sensitive to the representative strain rate. The yield stress increases with the strain rate. The highest the yield stress, the higher the elastic part of the representative strain (for a constant Young modulus). Since the total representative strain is constant, its plastic part decreases when the velocity increases.

The behavior model gives a logarithmic proportionality between the representative stress and the representative strain rate. As a consequence, Eq. (20) can be written thanks to Eq. (16):

$\Re \propto \frac{\ln \left(\dot{\varepsilon}_{r}^{e}\right)}{E a_{c} / R} \propto \frac{\ln (V)}{E a_{c} / R}$

Eq. (21) is suitable whatever the value of the Young modulus. However, the higher the Young modulus, the lower the recovery. Fig. 11 shows that at a fixed value of velocity the recovery decreases when the Young modulus increases. However, when the Young modulus increases the level of the representative stress corresponding to the beginning of the plasticity is early reached on the strain scale. Consequently the level of the reversible strain decreases. 


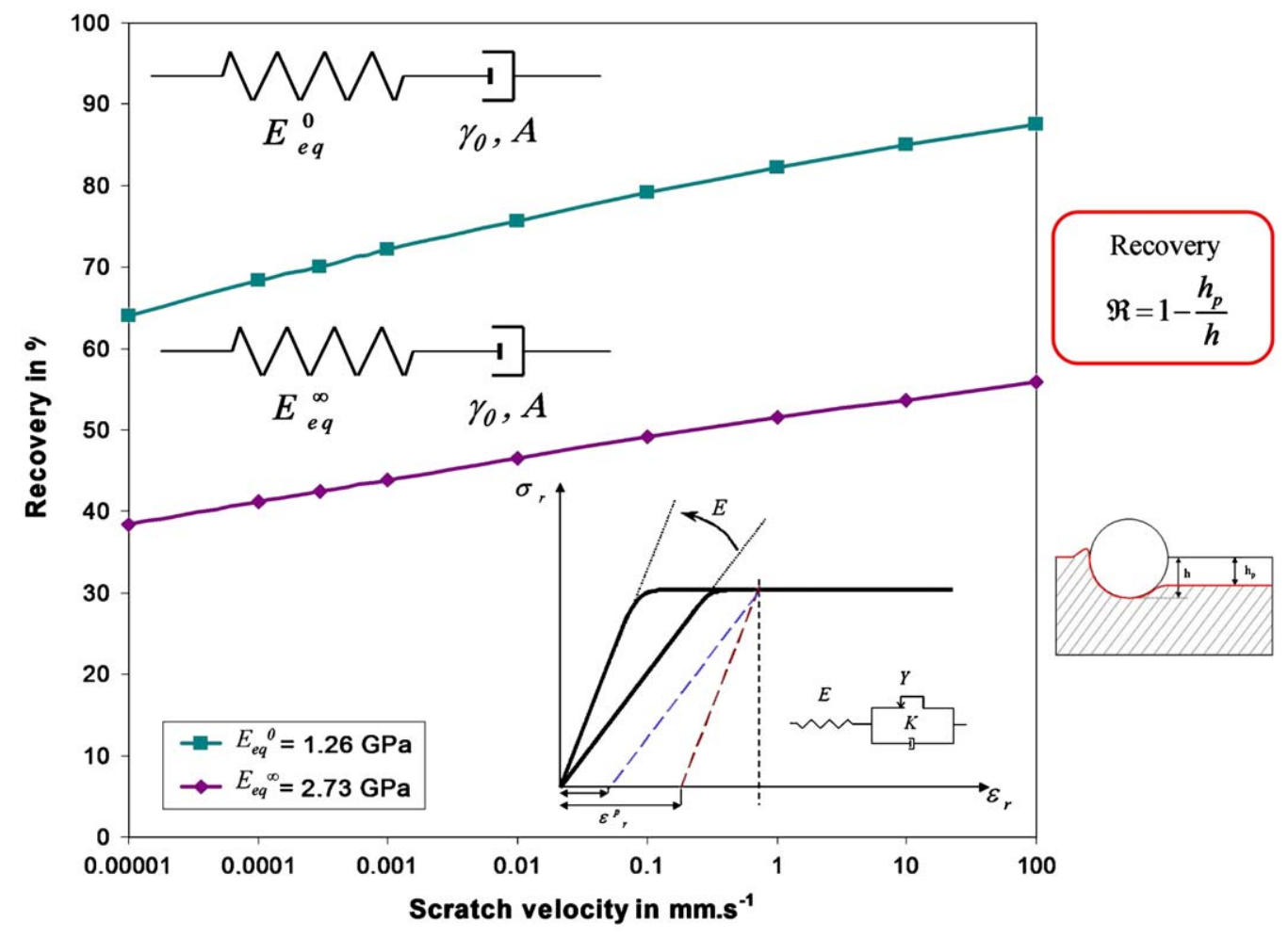

Fig. 11. Recovery vs. tip velocity for two different Young's moduli using the modified elastic-viscoplastic Arruda-Boyce model.

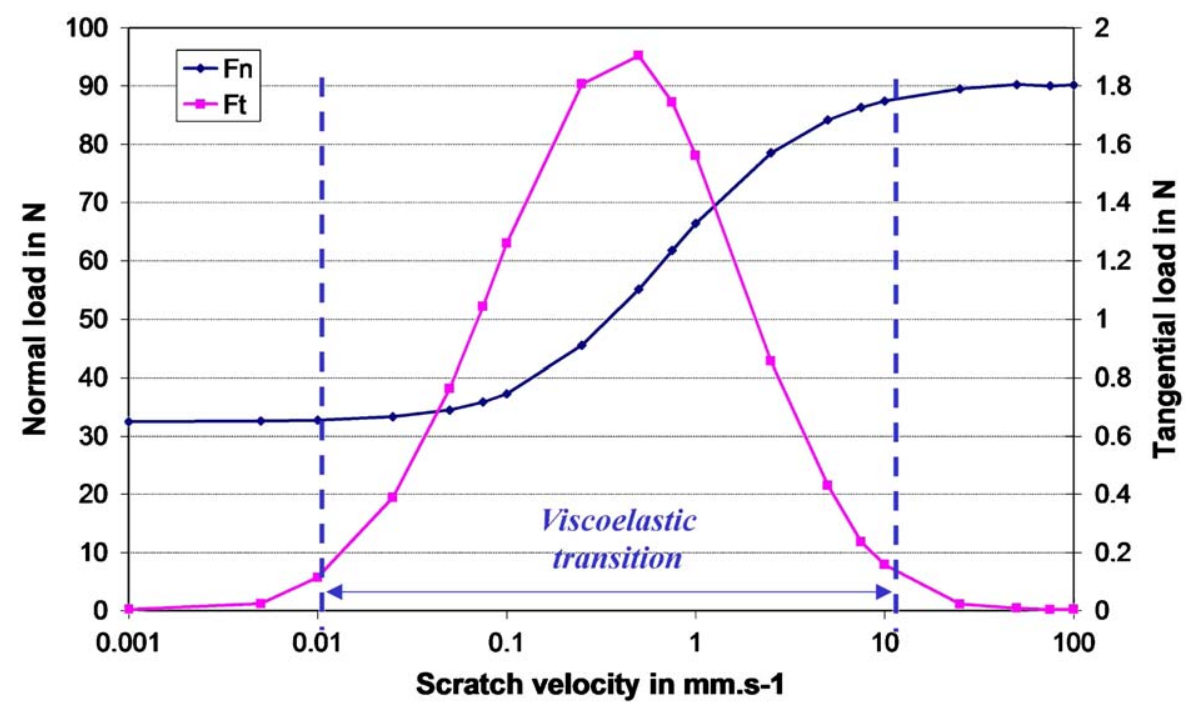

Fig. 12. Tangential and normal loads during a viscoelastic scratch.

\subsection{The viscoelastic transition}

The previous section shows the influence of the value of the Young modulus on the scratch response. This mechanical property of the polymers depends notably on the strain rate. We study here this aspect by using the viscoelastic model presented above.

Such a model leads to a perfectly reversible strain. The recovery is total that is why we pay attention to the variation of tangential and normal loads applied to the tip to keep the penetration and the velocity constant.

Fig. 12 shows two curves, one for the tangential load $F_{t}$ and the other for normal load $F_{n}$.
Since the penetration remains constant for all tip velocities, the load applied on the tip depends on the tip velocity.

For low tip velocities, the normal load $F_{n}$ curve is constant and the tangential load $F_{t}$ presents negligible values (frictionless contact). This state corresponds to a quasi-elastic response.

On the other side, for high tip velocities, $F_{n}$ has reached an higher constant value and the tangential load $F_{t}$ presents again negligible values. It is an other elastic response but defined by a higher stiffness than the previous one because of the higher value of $F_{n}$.

Between these two states, in a particular range of tip velocities the tangential load $F_{t}$ reaches a peak, whereas $F_{n}$ increases. This range of velocities corresponds directly to the one of the 
viscoelastic transition. The deformation of the material is strongly delayed and the indenter is submitted to a bigger tangential resistance. The deformation of the material is dissipative and depends strongly on the tip velocity.

Let us note that this range is very sharp because of the linear dependency of the viscous element on the strain rate. Polymers present a viscoelastic transition often wider than 10 decades of velocities. As a consequence, this result does not represent the behavior of a polymer but it shows clearly the influence of this type of model on the measurement of the scratch load.

\subsection{Viscoelasticity and recovery}

The viscoelastic and viscoplastic models are now connected to build up the complete model described above. This study aims at comparing the elastic-viscoplastic and viscoelastic-viscoplastic recoveries.

In Fig. 13, the two limit curves $E_{e q}^{0}$ and $E_{e q}^{\infty}$ related to the elastic-viscoplastic behavior, already analyzed, are also displayed. The third curve, called $\eta_{1}$, corresponds to the recovery due to the viscoelastic viscoplastic model.

This last curve presents two particularities: first, both ends of it are very close to the elastic-viscoplastic curves. At small tip velocities, the curve $\eta_{1}$ is close to the curve $E_{e q}^{0}$. This is explained by the fact that this modulus $\left(E_{e q}^{0}\right)$ is equal to the equivalent modulus of the Poynting model taken for the strain rates near zero. Such tip velocities are out of the range of the viscoelastic transition and the model behaves as an elastic one with an equivalent stiffness corresponding to small strain rates. For the same reason, the curves $\eta_{1}$ and $E_{e q}^{\infty}$ coincide at high tip velocities since $E_{e q}^{\infty}$ is the equivalent modulus of the Poynting model.

Between these two sections, in the particular range of velocities where the viscoelastic transition takes place, the curve $\eta_{1}$ decreases dramatically. In this range the equivalent modulus increases with the tip velocity and, as it has been shown previously, the recovery decreases when the stiffness increases.
To account for the dependency of the stiffness on the velocity Eq. (21) can be written as :

$\Re \propto \frac{\ln (V)}{E(V) a_{c} / R}$

When the viscoelastic transition is sharp, that is to say when the variation of the stiffness is important in a sharp range of frequencies, the influence of the stiffness on the recovery is higher than the one of the tip velocity. That happens here, but the viscoelastic model does not correspond to the one of polymers. As a conclusion, the ratio $\ln (V) / E(V)$ drives the dependency of the recovery on the velocity.

These results show that the viscoelasticity of viscoelasticviscoplastic material, like amorphous polymers, can lead to an important variation of values measured in scratch test. This variation is even higher than the viscoelastic transition is sharper.

\section{Conclusions}

It is well known that amorphous polymers display complex viscoelastic-viscoplastic behavior. However, such materials are often modeled by using an elastic-viscoplastic behavior law when the strain level is enough to activate plastic deformation. In the particular case of the scratch test some study have been started in order to highlight the necessity to account for the viscoelasticity. The first step of this expensive and difficult task deals with the understanding of the effect of the viscoelastic behavior in scratch test thanks to a numerical approach. Three major steps have been developed in this way.

The first main result concerns the study of the elasticviscoplastic recovery. The particular model of Arruda-Boyce [17] has been implemented in the finite element code Systus ${ }^{\mathbb{R}}$. Several simulations of the ploughing process at a fixed penetration depth have been carried out for different tip velocities. This shows that the recovery increases with the tip velocity and for a fixed value of

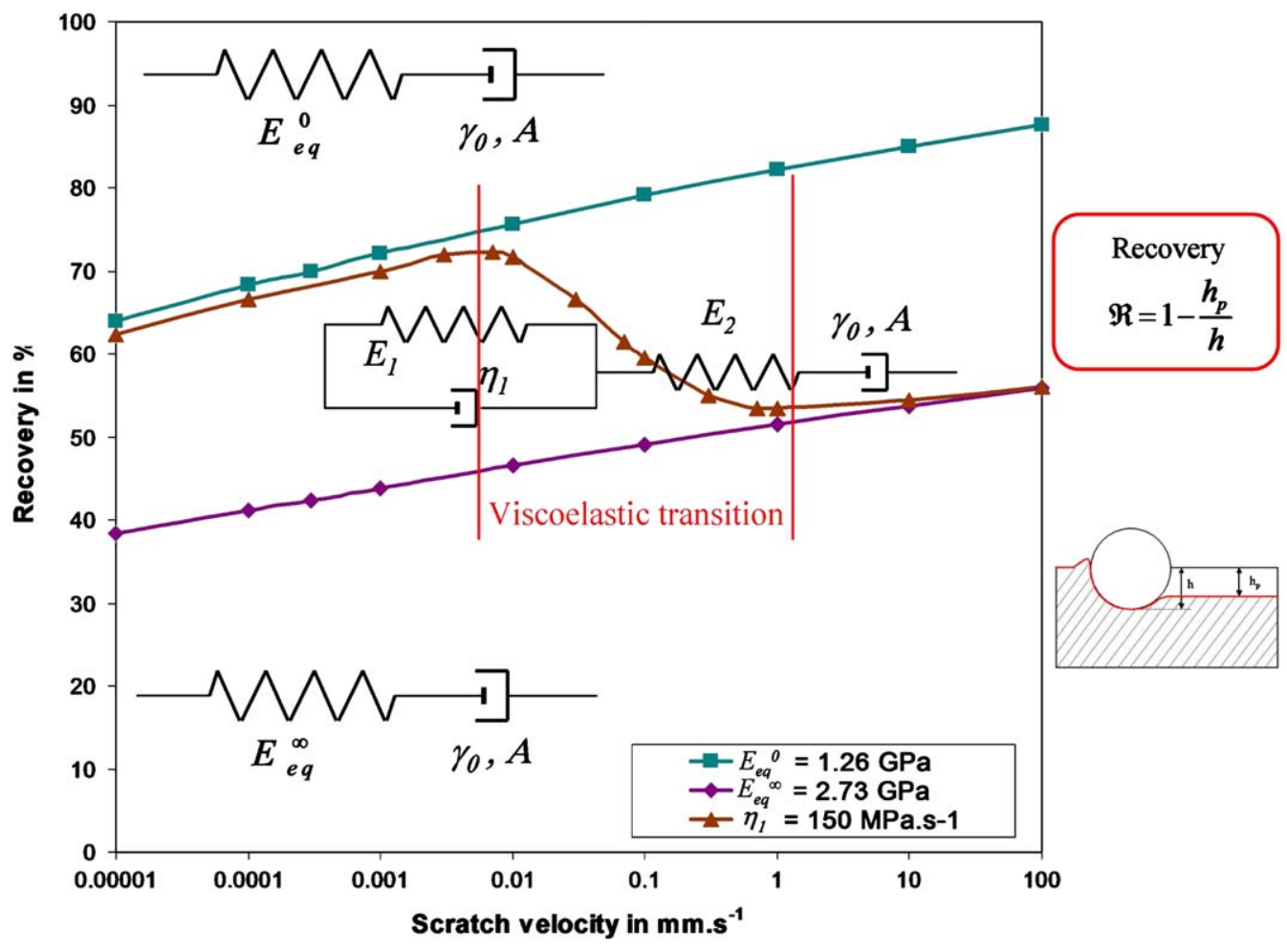

Fig. 13. Recovery in elastic-viscoplastic and viscoelastic-viscoplastic scratches. 
the velocity, the recovery depends directly on the value of the Young modulus.

Since the viscoelasticity is classically employed to model the variation of the stiffness with the strain rate, the second step of this study deals with the analysis of the normal load and the tangential load measured during scratch simulations at fixed penetration depth due to a purely viscoelastic behavior. In spite of the frictionless contact, the tangential load reaches a non-zero value describing a tangential resistance due to the delayed elastic response of the material. That takes place in the viscoelastic transition where the normal load increases because the equivalent stiffness modulus increases.

The viscoelastic transition leads to a particular response in scratch test, consequently the third part of this paper compares elastic-viscoplastic and viscoelastic-viscoplastic recoveries. To carry out viscoelastic-viscoplastic simulations we have developed a specific algorithm, the series model in the finite element code Systus ${ }^{\circledR}$, allowing to connect in series two models of any kind. As it has been shown, in the range of tip velocity corresponding to the viscoelastic transition, the viscoelastic-viscoplastic recovery decreases when the tip velocity increases. That corresponds to the increase of the equivalent stiffness of the model.

Forthcoming work will specifically address the problem of the viscoelasticity in modeling the mechanical behavior of amorphous polymers in scratch test.

\section{References}

[1] Wredenberg F. Per-Lennart Larsson. Scratch testing of metals and polymers: experiments and numerics. Wear 2009;266(1-2):76-83.

[2] Bucaille JL, Felder E, Hochstetter G. Mechanical analysis of the scratch test on elastic and perfectly plastic materials with the three-dimensional finite element modelling. Wear 2001;249:422-32.

[3] Barge M, Kermouche G, Gilles P, Bergheau J-M. Experimental and numerical study of the ploughing part of abrasive wear. Wear 2003:225:30-7.

[4] Bertrand-Lambotte P, Loubet JL, Verpy C, Pavan S. Understanding of automotive clearcoats scratch resistance. Thin Solid Films 2002;420421:281-6.

[5] Bucaille JL. Simulation numérique de l'indentation et de la rayure des verres organiques. PhD thesis, ENSM de Paris; 2001.

[6] Gauthier C, Lafaye S, Schirrer R. Elastic recovery of a scratch in a polymeric surface: experiments and analysis. Tribology International $2001 \cdot 34: 469-79$.

[7] Kermouche G, Aleksy N, Loubet JL, Bergheau JM. Finite element modeling of the scratch response of a coated time-dependent solid. Wear 2009;267:1945-53.
[8] Briscoe BJ, Evans PD, Pelillo E, Sinha SK. Scratching maps for polymers. Wear 1996;200:137-47.

[9] Jardret V, Zahouani H, Loubet JL, Mathia TG. Understanding and quantification of elastic and plastic deformation during a scratch test. Wear 1998;218:8-14.

[10] Kermouche G, Kaiser A-L, Gilles P, Bergheau J-M. Combined numerical and experimental approach of the impact-sliding wear of a stainless steel in a nuclear reactor. Wear 2007;263:1551-5.

[11] Koch T, Machl D. Evaluation of scratch resistance in multiphase pp blends. Polymer Testing 2007;26(7):927-36.

[12] Felder E, Bucaille JL. Mechanical analysis of the scratching of metals and polymers with conical indenters at moderate and large strains. Tribology International 2006;39:70-87.

[13] Gauthier C, Schirrer R. Time and temperature dependence of the scratch properties of poly(methylmethacrylate) surfaces. Journal of Materials Science 2000;35:2121-30.

[14] G'Sell C, Haudin JM. Introduction à la mécanique des polymères, Institut National Polytechnique de Lorraine, 1995.

[15] Drozdov AD. A constitutive model in viscoelastoplasticity of glassy polymers. Polymer 1999;40:3711-27.

[16] Bardenhagen SG, Stout MG, Gray GT. Three-dimensional, finite deformation, viscoplastic constitutive models for polymeric materials. Mechanics of Materials 1997;25:235-53.

[17] Arruda EM, Boyce MC. Evolution of plastic anisotropy in amorphous polymers during finite straining. International Journal of Plasticity 1993;9:697-720.

[18] Arruda EM, Boyce MC. Effects of initial anisotropy on the finite strain deformation behavior of glassy polymers. International Journal of Plasticity 1993;9:783-811.

[19] Hasan OA, Boyce MC. A constitutive model for the non linear viscoelastic viscoplastic behavior of glassy polymers. Polymer Engineering and Science 1995;35:331-44.

[20] Argon AS. A theory for the low temperature plastic deformation of glassy polymers. Philosophical Magazine 1973;28:839-65.

[21] Ward IM. Mechanical properties of solid polymers. New York: Wiley; 1979

[22] Systus/Sysweld. User's manual, 2006.

[23] Belytschko T, Kam Liu W, Moran B. Non linear finite elements for continua and structures. New York: Wiley; 2000.

[24] Tabor D. The hardness of solids. Review of Physics in Technology 1970;1:145-79.

[25] Pelletier H, Durier A-L, Gauthier C, Schirrer R. Viscoelastic and elastic-plastic behaviors of amorphous polymeric surfaces during scratch. Tribology International 2008;41:975-84.

[26] Johnson KL. The correlation of indentation experiments. Journal of the Mechanics and Physics of Solids 1970;18:115-26.

[27] Bucaille JL, Felder E. Finite-element analysis of deformation during indentation and scratch tests on elastic-perfectly plastic materials. Philosophical Magazine 2002;82:2003-12.

[28] Kermouche G, Loubet JL, Bergheau JM. An approximate solution to the problem of cone or wedge indentation of elastoplastic solids. Comptes Rendus Mecanique 2005;333(5):389-95.

[29] Kermouche G, Loubet J-L, Bergheau J-M. Extraction of stress-strain curves of elastic-viscoplastic solids using conical/pyramidal indentation testing with application to polymers. Mechanics of Materials 2008;40(4-5):271-83. 